KOMPASS

Dermatologie

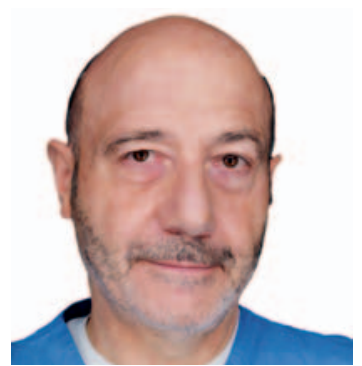

\section{Pierre Foss}

Niedergelassener Facharzt für Hautund Geschlechtskrankheiten, Wadern
Sieben Fragen an

\section{Dr. Pierre Foss}

\section{Träger des Innovationspreises Dermatologie 2017}

\section{Forschungsschwerpunkt}

- Dermatologische Zytologie und fotografische Dokumentation in der Dermatologie (DOCUDERM-Projekt)

\section{Akademischer Werdegang}

- Niedergelassener Facharzt für Haut- und Geschlechtskrankheiten, Wadern (Saarland)

- Weiterbildung zum Facharzt für Haut- und Geschlechtskrankheiten an der Klinik für Dermatologie, Venerologie und Allergologie, Homburg

- Patente zur Abformmikroskopie der Haut und zur Analyse der Haut und der Nägel über die Fräszytologie

- Nominierung zum Innovationspreis 2016 mit dem Projekt «Energieautarke Hautarztpraxis»

- Stipendiat der Deutschen Forschungsgemeinschaft (DFG)

- Ausbildung zum Psychiater im ehemaligen Landeskrankenhaus Merzig.

Was ist das Ziel Ihres ausgezeichneten Projekts «Zytoderm»?

Ziel des innovativen Projekts «Zytoderm» ist, zytologische Diagnostik routinemäßig in den dermatologischen Alltag einzuführen. Diese diagnostischen Maßnahmen sollten zukünftig auch entsprechend abgerechnet werden können. Die Voraussetzung dafür ist, dass die Kolleginnen und Kollegen aus der Dermatologie entsprechende Fortbildungen absolvieren und eventuell zukünftig eine Zertifizierung oder eine Qualitätssicherung erhalten und ihre Kenntnisse nachweisen können.

\section{Wie schätzen Sie den momentanen Stand der zytologischen Diagnostik in der Dermatologie ein?}

Nach meiner Rede zur Preisverleihung des Innovationspreises Dermatologie 2017 durch den Berufsverband der Deutschen Dermatologen (BVDD) in Frankenthal hat mich die positive Resonanz der Dermatologen sehr überrascht. Die ausgelegten Listen, in denen sich die Kolleginnen und Kollegen eintragen konnten, um weitere Informationen über die dermatologische Zytologie zu erhalten, haben nicht ausgereicht. Ich habe nach Gesprächen doch deutlich erkennen müssen, dass die dermatologische Zytologie momentan nur rudimentär, z.B. als Abriss-Zytologie mithilfe des Tesafilms, genutzt wird. Die von mir schon 2008 veröffentlichte Wundgrundzytologie im Sinne einer Bürstenabstrich-Zytologie wird so gut wie gar nicht eingesetzt, ebenso auch nicht die von mir erfundene und patentierte Fräszytologie der Haut und Nägel. Es bleibt also noch viel zu tun für die Fortbildung der Kollegen, um diese einfachen, aber nützlichen Untersuchungsmethoden im Fachbereich Dermatologie zu verankern. Das kann ich jedoch nicht alleine leisten.

\section{KARGER}

Fax +497614520714 information@karger.com www.karger.com (c) 2018 S. Karger GmbH, Freiburg
Dr. Pierre Foss

Kräwigstraße 8

66687 Wadern, Deutschland

info @hautarzt-foss.de 
Worin liegt die Relevanz Ihrer Ergebnisse für die praktische

klinische Arbeit eines Dermatologen?

Seit 30 Jahren beschäftige ich mich mit zytologischen Untersuchungen der Haut. Aus meiner Sicht ist deren Bedeutung für die praktische Arbeit der Dermatologen sehr groß.

Ich selbst führe täglich etwa 20-40 solcher Untersuchungen durch. Sie gehört deshalb für mich zur Routinediagnostik in der Abklärung verschiedenster Hautkrankheiten. An erster Stelle sind hier vor allen Dingen die Infektionskrankheiten zu nennen wie z.B. bakterielle und mykotische Infektionen der Haut sowie die Wundgrundzytologie zur Beurteilung des bakteriellen Infektionsstatus von Wunden oder deren Reife- und Abwehrstatus. Hinzukommen auch Abgrenzungen von entzündungsbedingten Hautkrankheiten und letztendlich auch die bessere Diagnostik von Malignomen der Haut. Ein weiteres diagnostisches Feld ist die Hilfestellung der Zytologie bei der Dermatoskopie (z.B. Naevus bleu differentialdiagnostisch noduläres malignes Melanom, seborrhoische Keratose differentialdiagnostisch aktinische Keratose und Lentigo maligna).

In der Dermatologie kann die Zytologie im Vergleich zur Gynäkologie 10-20 Mal häufiger eingesetzt werden. Dies hat damit zu tun, dass die Haut sehr gut zugänglich ist und viele Hautkrankheiten sich oberflächlich bzw. in einfach zugänglichen Schichten der Epidermis abspielen, die nur eine mittlere Dicke von 0,1 mm hat.

\section{Wie sehen Sie die zytologischen Untersuchungen in Bezug zu anderen Fachgruppen?}

Vor allem bei der Detektion möglicher Malignome können die bewährten Beurteilungsparameter der Gynäkologen bei Zervixkarzinomen auch dermatologisch gut angewandt werden.
Wie haben Sie das Preisgeld von 5000 EUR verwendet? Ich habe dieses Geld meiner Frau geschenkt.

Womit werden Sie sich in naher Zukunft beschäftigen? Ich werde versuchen noch weitere Wissenschaftler in das «Zytoderm»-Projekt zu integrieren, um einen Ausbildungs- bzw. Fortbildungskatalog zu erstellen. Das Projekt ist jedoch auf Jahrzehnte hin ausgelegt und wird sicherlich meine Lebenszeit überdauern.

Womit schaffen Sie sich selbst und Ihrem Umfeld einen Ausgleich zum Einsatz in der Forschung?

Ich bin Gitarrist, Sänger, Poet und Künstler. Ich habe eigene Kunstformen wie die ARBO-ART, MYKO-ART und ROSTIS entwickelt, die ich teilweise als Ölbilder darstelle und auch auf Ausstellungen präsentiere. Zusätzlich gehört das Singen, Tanzen und der Sport selbstverständlich zu meinen bewährten Ausgleichsmaßnahmen.

Dr. Foss, herzlichen Dank für das Interview!

\section{ANGABEN ZUM PREIS}

Bereits zum 7. Mal zeichnete der Berufsverband der Deutschen Dermatologen (BVDD) eine zukunftsweisende Idee aus dem Bereich der fachärztlichen Tätigkeit aus. Mit der Auszeichnung fördert der BVDD gemeinsam mit der Professor-Paul-GersonUnna-Akademie Projekte, die den Behandlungserfolg von Patienten in der dermatologischen Praxis verbessern können. Der Preis wird jährlich vergeben und ist mit 5000 EUR dotiert. 